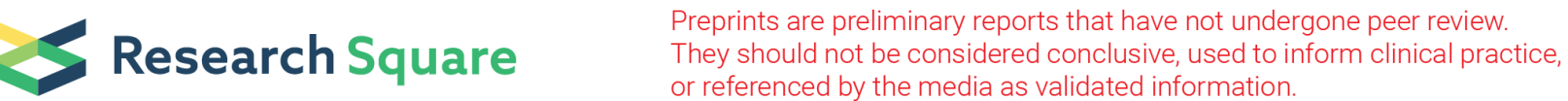

\section{Development and Validation for Prognostic Nomogram of Epithelial Ovarian Cancer Recurrence based on Circulating Tumor Cells and Epithelial- Mesenchymal Transition}

\section{Jiani Yang}

Shanghai Jiao Tong University School of Medicine Affiliated Renji Hospital

Jun Ma

Shanghai Jiao Tong University School of Medicine Affiliated Renji Hospital

Yue Jin

Shanghai Jiao Tong University School of Medicine Affiliated Renji Hospital

\section{Shanshan Cheng}

Shanghai Jiao Tong University School of Medicine Affiliated Renji Hospital

\section{Shan Huang}

Shanghai Jiao Tong University School of Medicine Affiliated Renji Hospital

\section{Nan Zhang}

Shanghai Jiao Tong University School of Medicine Affiliated Renji Hospital

Yu Wang ( $\nabla$ renjiwangyu@126.com )

Shanghai Jiao Tong University School of Medicine Affiliated Renji Hospital

\section{Research}

Keywords: circulating tumor cell, epithelial-mesenchymal transition, epithelial ovarian cancer, recurrence, risk stratification

Posted Date: November 11th, 2020

DOl: https://doi.org/10.21203/rs.3.rs-86051/v2

License: (c) (i) This work is licensed under a Creative Commons Attribution 4.0 International License. Read Full License

Version of Record: A version of this preprint was published at Scientific Reports on March 22nd, 2021. See the published version at https://doi.org/10.1038/s41598-021-86122-4. 


\section{Abstract}

We aimed to determine prognosis value of circulating tumor cells(CTCs) undergoing epithelialmesenchymal transition(EMT) in epithelial ovarian cancer(EOC) recurrence. We used CanPatrol CTCenrichment technique to detect CTCs from blood samples and classify subpopulations into epithelial, mesenchymal and hybrids. To construct nomogram, prognostic factors were selected by Cox regression analysis. Risk stratification was performed through Kaplan-Meier analysis among training group $(n=114)$ and validation group $(n=38)$. By regression screening, both CTC counts $(H R 1.187 ; 95 \% \mathrm{Cl} 1.098-1.752$; $\mathrm{p}=0.012)$ and $\mathrm{M}-\mathrm{CTC}(\mathrm{HR} 1.098 ; 95 \% \mathrm{Cl} 1.047-1.320 ; \mathrm{p}=0.009)$ were demonstrated as independent factors for recurrence. Other variables including pathological grade, FIGO stage, lymph node metastasis, ascites and CA-125 were also collected $(\mathrm{p}<0.005)$ to construct nomogram. The $\mathrm{C}$-index of internal and external validation for nomogram was 0.913 and 0.874 . We found significant predictive value for nomogram with/without CTCs (AUC 0.8705 and 0.8097). Taking CTC counts and M-CTC into separation, the values were 0.8075 and 0.8262 . Finally, survival curves of risk stratification based on CTC counts $(p=0.0241), M-$ CTC $(p=0.0107)$ and the nomogram $(p=0.0021)$ were drawn with significant difference. In conclusion, CTCs could serve as a novel factor for EOC prognosis. Nomogram model constructed by CTCs and other clinical parameters could predict EOC recurrence and perform risk stratification for clinical decisionmaking.

Trial registration: Chinese Clinical Trial Registry, ChiCTR-DDD-16009601, October 25, 2016

\section{Introduction}

Ovarian cancer was a leading cause of death among gynecological cancers, with 21,750 new cases and 13,940 deaths estimated for 2020 in the United State ${ }^{1}$. Due to the lack of early symptoms and physical signs, over $70 \%$ patients are diagnosed as advanced stages, resulting to the poor prognosis with a 10year survival rate of $5-21 \%^{2}$. Despite development in treatment techniques, approximately $80 \%$ of epithelial ovarian cancer (EOC) patients will suffer cancer recurrence after the primary treatment of standard cytoreductive surgery followed by adjuvant platinum-based chemotherapy ${ }^{3}$.

Therefore, effective methods for predicting EOC prognosis is of clinical significance to improve survival. The circulating tumor cells (CTCs), originating from solid tumors, are related to the hematogenous metastasis of various carcinomas, such as breast, prostate and ovarian cancer ${ }^{4-6}$. CTCs disseminate to distant sites through phenotypic changes, including epithelial-mesenchymal transition (EMT) that could help them to penetrate blood vessel ${ }^{7}$. In hepatocellular carcinoma, Qi LN et al. ${ }^{8}$ demonstrated that the epithelial-to-mesenchymal-CTC ratio was significantly associated with cancer recurrence and progression. Thus, apart from CTC counts, mesenchymal-CTC (M-CTC) percentage also has clinical relevance as a minimal-invasive approach to predict cancer recurrence and guide clinical therapy ${ }^{8,9}$.

Recently, CTC detection and isolation based on physical property have been applied in various solid tumors $6,10,11$. However, these approaches might fail to classify aggressive CTC subpopulations that 
undergo the EMT process. In this study, we used the CanPatrol CTC-enrichment technique based on the RNA in situ hybridization (RNA-ISH) to identify and classify all CTC subpopulations including epithelial, mesenchymal, and epithelial/mesenchymal hybrids with high efficiency ${ }^{12}$. This technique has been used in a range of carcinomas to predict prognosis ${ }^{8,13}$. However, to the best of our knowledge, this is the first prospective study to classify the prognosis value CTC of subpopulations undergoing EMT in EOC through the CanPatrol CTC-enrichment approach.

Moreover, given the poor prognosis of EOC, an effective risk stratification system is of great importance for clinicians in therapeutic decision-making process ${ }^{14}$. So, we aimed to construct the nomogram, a comprehensive model with graphical representation that could evaluate numerical probability of cancer recurrence for individual ${ }^{15}$. Most previous prognosis models were constructed based on general factors such as clinical stage, pathological grade, tumor histology and CA-125, with limited predictive value $\mathrm{e}^{16,17}$. So, the objective of our prospective study was to construct and validate the prognosis nomogram based on CTCs, more accurately as compared to current models in practice. By using this nomogram for risk stratification, we hope to develop a prediction tool, which could support therapeutic decision-making and might consequently improve prognosis of EOC patients.

\section{Results}

\section{Clinicopathological characteristics}

Demographic and clinicopathological features of all the EOC patients, including the training group $(n=114)$ and validated group ( $n=38)$, were listed in Table 1 and Table 2. Patients with early stage (FIGO I or II) and advanced stage (FIGO III or IV) accounted for 39(25.66\%) and 113(74.34\%), respectively. Patients diagnosed as low pathological grade (G1-G2) and high pathological grade (G3) accounted for $51(33.55 \%)$ and $101(66.45 \%)$ of all patients involved. There were $72(47.37 \%)$ patients with histologyproved lymph node metastasis, and $58(38.16 \%)$ cases presented with ascites. The mean value ( \pm SD) of CTC counts, M-CTC percentage and CA-125 was $8.70 \pm 5.69,0.24 \pm 0.19$, and $990.71 \pm 365.41(\mathrm{U} / \mathrm{mL})$, respectively. The median time of follow-up was 30 months (range, 24-35 months). The investigated clinical data of patients from both groups were analyzed and no significant difference were found, indicating no selection bias ( $p$-value $>=0.05$ ).

Table 1. Baseline clinicopathological characteristics of the 152 EOC patients involved grouped by training set and validation set 


\begin{tabular}{llllc}
\hline Variables & Total (n=152) & Training group (n=114) & Validated group (n=38) & p-value \\
\hline Age (years) & $58.06 \pm 9.47$ & $57.81 \pm 10.25$ & $58.10 \pm 9.73$ & 0.879 \\
\hline BMI (kg/m2) & $22.86 \pm 0.73$ & $22.91 \pm 0.47$ & $22.85 \pm 0.93$ & 0.604 \\
\hline Menopausal status, $\mathrm{n}(\%)$ & & & & 0.924 \\
\hline Pre/peri-menopause & $65(42.76 \%)$ & $49(32.24 \%)$ & $16(10.53 \%)$ & - \\
\hline Post-menopause & $87(57.24 \%)$ & $65(42.76 \%)$ & $22(14.47 \%)$ & - \\
\hline Fertility history, $\mathrm{n}$ (\%) & & & & 0.852 \\
\hline 0-1 & $82(53.95 \%)$ & $62(40.79 \%)$ & $20(13.16 \%)$ & - \\
\hline$\geq 2$ & $70(46.05 \%)$ & $52(34.21 \%)$ & $18(11.84 \%)$ & - \\
\hline Tumor size (cm) & $7.26 \pm 3.15$ & $6.39 \pm 4.62$ & $7.37 \pm 3.21$ & 0.285 \\
\hline Pathological grade, $\mathrm{n}$ (\%) & & & & 0.765 \\
\hline G1-2 & $51(33.55 \%)$ & $39(25.66 \%)$ & $12(7.89 \%)$ & - \\
\hline G3 & $101(66.45 \%)$ & $75(49.34 \%)$ & $26(17.11 \%)$ & - \\
\hline FIGO stage, $\mathrm{n}$ (\%) & & & & 0.748 \\
\hline I-II & $39(25.66 \%)$ & $30(19.74 \%)$ & $9(5.92 \%)$ & - \\
\hline III-IV & $113(74.34 \%)$ & $84(55.26 \%)$ & $29(19.08 \%)$ & - \\
\hline Lymph node metastasis, $\mathrm{n}(\%)$ & $72(47.37 \%)$ & $52(34.21 \%)$ & $20(13.16 \%)$ & 0.453 \\
\hline Ascites, $\mathrm{n}$ (\%) & $58(38.16 \%)$ & $43(28.29 \%)$ & $15(9.87 \%)$ & 0.847 \\
\hline Histological type, $\mathrm{n}$ (\%) & & & & 0.954 \\
\hline Serous & $92(60.53 \%)$ & $69(45.39 \%)$ & $23(15.13 \%)$ & - \\
\hline Mucinous & $24(15.79 \%)$ & $18(11.84 \%)$ & $6(3.95 \%)$ & - \\
\hline Endometrioid & $17(11.18 \%)$ & $12(7.89 \%)$ & $5(3.29 \%)$ & - \\
\hline Others & $19(12.50 \%)$ & $15(9.87 \%)$ & $4(2.63 \%)$ & - \\
\hline
\end{tabular}

Table 2. Clinical features of the 152 EOC patients involved grouped by training set and validation set

\begin{tabular}{llllc}
\hline Variables & Total $(\mathrm{n}=152)$ & Training group $(\mathrm{n}=114)$ & Validated group $(\mathrm{n}=38)$ & $\mathrm{p}$-value \\
\hline CTC counts (n) & $8.70 \pm 5.69$ & $8.64 \pm 5.38$ & $8.72 \pm 4.65$ & 0.935 \\
\hline M-CTC ( $\%)$ & $0.24 \pm 0.19$ & $0.25 \pm 0.21$ & $0.22 \pm 0.14$ & 0.413 \\
\hline Neutrophil (10^9/L) & $5.22 \pm 2.91$ & $4.67 \pm 2.83$ & $5.31 \pm 1.96$ & 0.198 \\
\hline lymphocyte (10^9/L) & $1.42 \pm 0.63$ & $1.46 \pm 0.85$ & $1.37 \pm 0.77$ & 0.564 \\
\hline Platelet (10^9/L) & $320.39 \pm 78.68$ & $299 \pm 73.94$ & $314 \pm 80.21$ & 0.291 \\
\hline Albumin (g/L) & $40.49 \pm 6.58$ & $38.27 \pm 9.38$ & $41.22 \pm 10.02$ & 0.101 \\
\hline CA-125 (U/mL) & $990.71 \pm 365.41$ & $1001.23 \pm 330.98$ & $986.53 \pm 310.27$ & 0.810 \\
\hline CA-199 (U/mL) & $129.03 \pm 53.18$ & $121.88 \pm 48.27$ & $132.5 \pm 59.12$ & 0.270 \\
\hline AFP (ng/ml) & $6.21 \pm 4.78$ & $6.43 \pm 5.99$ & $5.93 \pm 3.45$ & 0.627 \\
\hline CEA (ng/ml) & $3.02 \pm 2.57$ & $2.95 \pm 2.60$ & $3.21 \pm 2.47$ & 0.589 \\
\hline HE4 (pmol/l) & $536.12 \pm 54.48$ & $542.32 \pm 79.39$ & $521.39 \pm 62.10$ & 0.141 \\
\hline
\end{tabular}

\section{Univariable and multivariate regression analysis of training group}

Figure 1 showed that patients suffered cancer recurrence had higher CTC counts and M-CTC percentage $(p$-value $<0.05)$. To further determine the independent predictive indexes, univariate and multivariate 
analyses were performed (Table 3). In the univariable COX regression analysis, parameters including age, tumor size, menopausal status, pathological grade, FIGO stage, lymph node metastasis, ascites, CTC counts, M-CTC percentage, albumin level, CA-125 and HE4 were significantly associated with ovarian cancer recurrence. Then, these indicators were included into the multivariate Cox hazards model for further analysis. The results demonstrated that pathological grade (HR, 1.382; 95\% Cl, 1.104-3.965; $p=$ $0.041)$, FIGO stage (HR, 2.391; $95 \% \mathrm{Cl}, 1.230-4.377 ; \mathrm{p}=0.011)$, lymph node metastasis $(\mathrm{HR}, 1.312 ; 95 \% \mathrm{Cl}$, 1.029-2.975; $p=0.039)$, ascites (HR, 1.215; 95\% Cl, 1.067-1.806; $p=0.026)$, CTC counts (HR, 1.187; 95\% $\mathrm{Cl}, 1.098-1.752 ; \mathrm{p}=0.012)$, M-CTC percentage (HR, 1.098; 95\% Cl, 1.047-1.320; $\mathrm{p}=0.009)$ and CA-125 (HR, $1.097 ; 95 \% \mathrm{Cl}, 1.021-1.373 ; \mathrm{p}=0.028$ ) were independent prognostic factors for OS of EOC patients (Table $3)$.

Table 3. Clinicopathological features selection using the univariable and multivariate Cox logistic regression model among the training group

\begin{tabular}{|c|c|c|c|c|c|c|}
\hline \multirow[t]{2}{*}{ Variables } & \multicolumn{3}{|c|}{ Univariate analysis } & \multicolumn{3}{|c|}{ Multivariate analysis } \\
\hline & Hazard Ratio & $95 \% \mathrm{CI}^{*}$ & P-value & Hazard Ratio & $95 \% \mathrm{CI}^{*}$ & P-value \\
\hline Age (years) & 1.131 & $1.028-1.954$ & 0.045 & 1.089 & $0.922-1.317$ & 0.058 \\
\hline BMI (kg/m2) & 1.016 & $0.969-1.064$ & 0.514 & - & - & - \\
\hline Tumor size $(\mathrm{cm})$ & 1.271 & $1.014-1.298$ & 0.048 & 0.933 & 0.815-1.069 & 0.314 \\
\hline \multicolumn{7}{|l|}{ Menopausal status } \\
\hline Pre/peri-menopause & Reference & - & - & Reference & - & - \\
\hline Post-menopause & 1.153 & $1.037-1.209$ & 0.047 & 3.498 & $0.196-6.374$ & 0.394 \\
\hline \multicolumn{7}{|l|}{ Fertility history } \\
\hline $0-1$ & Reference & - & - & - & - & - \\
\hline$\geq 2$ & 0.989 & $0.967-1.025$ & 0.574 & - & - & - \\
\hline \multicolumn{7}{|l|}{ Pathological grade } \\
\hline G1-2 & Reference & - & - & Reference & - & - \\
\hline G3 & 1.509 & $1.031-4.506$ & 0.034 & 1.382 & $1.104-3.965$ & 0.041 \\
\hline \multicolumn{7}{|l|}{ FIGO stage } \\
\hline I-II & Reference & - & - & Reference & - & - \\
\hline III-IV & 2.534 & $1.293-4.966$ & 0.007 & 2.391 & $1.230-4.377$ & 0.011 \\
\hline Lymph node metastasis & 1.269 & $1.045-3.582$ & 0.036 & 1.312 & $1.029-2.975$ & 0.039 \\
\hline Ascites & 1.123 & $1.042-1.301$ & 0.017 & 1.215 & $1.067-1.806$ & 0.026 \\
\hline CTC counts (n) & 1.241 & $1.108-1.384$ & 0.009 & 1.187 & $1.098-1.752$ & 0.012 \\
\hline M-CTC (n \%) & 1.147 & $1.051-1.288$ & 0.006 & 1.098 & $1.047-1.320$ & 0.009 \\
\hline Neutrophil $\left(10^{\wedge} 9 / \mathrm{L}\right)$ & 1.015 & $0.992-1.038$ & 0.207 & - & - & - \\
\hline lymphocyte (10^9/L) & 1.066 & $0.888-1.278$ & 0.494 & - & - & - \\
\hline Platelet $\left(10^{\wedge} 9 / \mathrm{L}\right)$ & 1.002 & $0.997-1.007$ & 0.351 & - & - & - \\
\hline Albumin (g/L) & 0.909 & $0.849-0.972$ & 0.025 & 1.027 & $0.980-1.077$ & 0.266 \\
\hline $\mathrm{CA}-125(\mathrm{U} / \mathrm{mL})$ & 1.101 & $1.002-1.203$ & 0.019 & 1.097 & $1.021-1.373$ & 0.028 \\
\hline CA-199 (U/mL) & 0.977 & $0.942-1.012$ & 0.198 & - & - & - \\
\hline $\operatorname{AFP}(\mathrm{ng} / \mathrm{ml})$ & 1.001 & $0.963-1.040$ & 0.958 & - & - & - \\
\hline CEA (ng/ml) & 1.001 & 0.999-1.012 & 0.805 & - & - & - \\
\hline HE4 (pmol/l) & 1.217 & $1.104-1.232$ & 0.039 & 0.959 & $0.850-1.081$ & 0.489 \\
\hline
\end{tabular}




\section{Construction of EOC recurrence nomogram}

The clinicopathological parameters (FIGO stage, pathological grade, lymph node metastasis, ascites, CTC counts, M-CTC percentage and CA-125) were selected by both univariable and multivariate Cox logistic regression were channeled into construction of the nomogram (Figure. 2A), while a nomogram without CTC counts and M-CTC percentage were also constructed for comparation (Figure. 2B). In the training group, the C-index of 1000 sample bootstrap was 0.913 and 0.832 for the nomogram with and without CTCs. When applied to the validation cohort, the C- index was 0.874 and 0.782 , respectively, which showed significant prognosis value of discrimination in both cohorts for the nomogram with CTC counts and M-CTC percentage.

Further risk stratification in EOC patients calibration curves manifested that the probability of predicted 1year and 2-year recurrence rate in nomogram were well consistent between the predicted outcome of cancer recurrence and actual observation in the training group (Figure. 3A and 3B). Moreover, in the external validation group, the calibration curves also illustrated good validation between predicted and observed 1- and 2-year recurrence proportions (Figure. 3C and 3D). The discrimination and calibration validation of external group definitely certificated that nomogram models in this study is comparatively accurate enough to predict the recurrence probability of patients with EOC.

\section{Risk stratification in EOC patients}

According to the ROC curve, the AUC values of CTC counts, M-CTC percentage and CA- 125 were 0.8073 , 0.8262 and 0.7735 , respectively (Figure. $4 \mathrm{~A}$ ). For the nomogram with/without CTC counts and M-CTC percentage, the AUC were 0.8705 and 0.8097 (Figure. 4B). Meanwhile, as illustrated in Figure. 4C and 4D, the discriminatory value of CTC counts and M-CTC percentage was significant among ovarian cancer patients, with the log-rank $P$ value of 0.0241 and 0.0107 , respectively. When stratified by CTC counts, patients with CTCs $>=9$ and $5<=$ CTCs $<9$ were associated with a 1.98 -fold increase $(95 \% \mathrm{Cl}, 1.04-2.47)$ and 1.24-fold increase ( $95 \% \mathrm{Cl}, 1.07-2.29)$ of recurrence rate, comparing to those with $\mathrm{CTCs}<5$, while patients with M-CTC percentage $>=0.3$ and $0.1<=\mathrm{M}-\mathrm{CTC}<0.3$ were associated with a 2.10 -fold increase $(95 \% \mathrm{Cl}, 1.54-2.66)$ and 1.43 -fold increase $(95 \% \mathrm{Cl}, 1.14-2.53)$ of recurrence rate, comparing to those with $\mathrm{M}-\mathrm{CTC}<0.1$.

Moreover, the patients were then divided into three risk groups (low-, intermediate- and high-risk groups) based on the nomogram-predicted recurrence probabilities. For the nomogram without CTCs, when compared with the low-risk group, the high-risk and intermediate-risk groups were associated with a 2.37fold increase $(95 \% \mathrm{Cl}, 1.28-4.83)$ and 1.48 -fold increase $(95 \% \mathrm{Cl}, 1.17-2.64)$ in the risk of recurrence, with the $p$ value of 0.0010 (Figure. 4E). Meanwhile, in the CTCs based nomogram, the high-risk and intermediate-risk groups were associated with a 3.14 -fold increase $(95 \% \mathrm{Cl}, 1.16-4.50)$ and 1.86 -fold increase $(95 \% \mathrm{Cl}, 1.70-3.96)$ in the risk of recurrence, with the $\mathrm{p}$ value of 0.0002 (Figure. 4F). 


\section{Discussion}

The clinical value of CTCs is constantly growing, as they could serve precision-medicine-based treatment of EOC patients by stratifying those with potential high recurrence risk. In this prospective study, we developed and validated a novel nomogram based on CTCs and other clinicopathological variables to categorize EOC patients with respect to tumor recurrence. We also found that the presence of CTC subpopulations, especially the M-CTC percentage is associated with ovarian cancer recurrence. To our knowledge, this is the very first recurrence risk stratification developed for EOC patients especially refer to CTCs undergoing EMT.

Increasing evidence indicated that CTCs is an independent predictor for prognosis in various solid carcinoma, including breast cancer, prostate cancer and hepatocellular cancer. The breast cancer studies have demonstrated that patients with CTCs $<5$ per $7.5 \mathrm{~mL}$ blood would suffer shorter PFS (2.1 months vs 7.0 months, $p<0.001)^{18,19}$. In prostate cancer, CTCs is considered as an independent predictor of the overall survival rate among castration-resistant prostate cancer patients $(p<0.05)^{6}$. However, in regard to ovarian cancer, whether CTCs detection was associated with prognosis remains controversial ${ }^{10,20}$. Judson PL et al. ${ }^{20}$ characterized CTCs by immunomagnetic beads conjugated to epithelial markers followed with the microscopic evaluation refer to specific cytoplasmic staining and did not find significant correlation between CTCs and prognosis. In contrast, Poveda A et al. ${ }^{10}$ analyzed CTCs using the CellSearch system and concluded that elevated CTCs could impart unfavorable prognosis of ovarian cancer patients. Differences in isolation and characterization technique in previous studies make it difficult to combine conclusions in agreement ${ }^{21}$. So, the standardization of CTCs detection technique is of great importance. In our study, we revealed that CTC counts was an independent prognosis factor for ovarian cancer recurrence through both univariable and multivariable analyses using CanPatrol CTCenrichment technique System. The high sensitivity of the CanPatrol techinique might attributed to a simple filter-based separation method that might reduce CTC loss caused by the complicated washing and centrifugation process ${ }^{22}$.

Meanwhile, the routine approach of Cellsearch System used in previous studies might fail to detect CTCs undergoing EMT, since it only isolate CTCs by the only tumor epithelial cell expression of EpCAM ${ }^{11,22}$ and not mesenchymal ones without epithelial markers. Thus, we used the CanPatrol CTC-enrichment technique System to detect aggressive CTCs subpopulation that might have undergone EMT through various target sequences, including EpCAM, CD45, CK8/18/19, vimentin and Twist ${ }^{5}$. For hepatocellular carcinoma, a previous study concluded that M-CTC percentage $>=2 \%$ prior to operation was a novel predictor for early recurrence with the AUC $0.75(95 \% \mathrm{Cl}, 0.66-0.84)^{8}$, which was partly consistent with our finding that ovarian cancer patients with M-CTC percentage $>=0.3$ and $0.1<=\mathrm{M}-\mathrm{CTC}<0.3$ were associated with a 2.10 -fold increase and 1.43 -fold increase of recurrence rate, when compared to those with M-CTC $<0.1$. To the best of our knowledge, this is the first study to reveal the considerable clinical value of both CTC counts and M-CTC percentage in ovarian cancer prognosis. 
Moreover, we aimed to develop a predictive nomogram to help facilitate the risk triage of ovarian cancer recurrence. Besides the presence of CTCs, we also selected several routinely collected risk factors including pathological grade, FIGO stage, lymph node metastasis, ascites and CA-125 to construct the nomogram in training group ${ }^{23-25}$. The clinical relevance of our nomogram was demonstrated by its internal and external validation with the C-index of 0.913 and 0.874 , which indicated that our model included in CTCs could provide a more reliable predictive evaluation for ovarian cancer recurrence than previous studies ${ }^{25,26}$.

Nevertheless, we further performed risk stratification of EOC patients based on CTC counts, M-CTC percentage and points derived from the nomogram. All the risk stratification was well validated by survival analysis $(\mathrm{p}<0.05)$ with the AUC higher than 0.75 as well. According to risk stratification, especially by the nomogram, we could carry out individualized and targeted treatment to improve prognosis of ovarian cancer.

However, there are also some limitations of our study. Firstly, the prospective study enrolled a relatively small sample size of $152 \mathrm{EOC}$ patients in a single center, which might limit the accuracy of results. To overcome this problem, additional multi-center studies with larger sample size would be of great importance to further validate our results. Second, detection efficiency might be biased since the CanPatrol system is a filtration-based system, allowing small CTCs easily cross the barrier. Thus, other CTCs collection techniques might also be used to improve detection efficiency in future studies.

In conclusion, CTCs, especially those undergoing EMT hold promise prognostic value as minimallyinvasive biomarkers for ovarian cancer recurrence. By the advanced CanPatrol CTC-enrichment technique, our study evaluated both CTC counts and M-CTC percentage to clarify their clinical value. The prognostic nomogram based on CTCs and EMT could support clinical decision-making and provide cues for early intervention among EOC patients.

\section{Methods}

\section{Study design and patients}

We enrolled 181 patients with pathologically diagnosed EOC who underwent surgery at the Department of Obstetrics and Gynecology, Renji Hospital Affiliated to Shanghai Jiaotong University School of Medicine between June 2017 to October 2019. The criteria for inclusion in this study were: 1) newly diagnosis EOC confirmed by pathological biopsy; 2) no coexisting cancers or prior cancers within 5 years; 3) no preoperative treatment, including neoadjuvant chemotherapy or radiotherapy; The exclusion criteria were as follows: 1) lost to follow-up ( $n=9)$; 2 ) without detailed clinical, laboratory, imaging, and treatment data $(n=8) ; 3)$ underwent other treatments, such as radiotherapy or immunotherapy $(n=5) ; 4)$ without consent to use medical information for the research purpose $(n=4)$; and 5$)$ with status not allowing the treatment of operation followed by chemotherapy $(n=3)$. As a result, 152 patients were assessed in the analysis (Figure 5). 
In order to achieve optimal tumor debulking, the operation for all involved patients was aimed at maximal ovarian tumor resection without visible residual tumor. The surgery was followed by standardized paclitaxel and platinum chemotherapy. All patients were followed up until September 1st, 2020. This study was approved by ethics Committee of Renji Hospital Affiliated to Shanghai Jiaotong University School of Medicine and all involved subjects provided informed consents for use of their information on research purpose. All experiments were performed in accordance with relevant guidelines and regulations.

\section{Clinicopathological data collection}

The clinical stage was evaluated according to the International Federation of Gynecology and Obstetrics (FIGO) stage system. Routine blood tests and tumor marker measurements, including carbohydrate antigen-125 (CA-125), carbohydrate antigen-199 (CA-199), carcinoembryonic antigen (CEA), alpha fetoprotein (AFP), and human epididymis protein 4 (HE4) were conducted within 1 day before surgery. The clinicopathologic variables, including age, Body Mass Index (BMI), tumor size, menopausal status, fertility history, pathological grade, the FIGO stage, lymph node metastasis, ascites and histological type were reviewed from medical records. Disease-free survival (DFS) was measured from the date of surgery to the last follow-up visit or ovarian cancer recurrence, which was defined through the latest clinical evidence. The diagnosis of EOC recurrence was performed by at least two oncologists to avoid bias.

\section{Isolation and characterization of CTCs}

Peripheral blood samples ( $5 \mathrm{~mL}$, anticoagulated with EDTA) were collected 1 day before treatment, stored at $2-8^{\circ} \mathrm{C}$ and processed within 4 hours after sampling ${ }^{7}$. To avoid potential skin cell contamination caused by venipuncture, the first $2 \mathrm{~mL}$ of blood should be discarded ${ }^{27}$.

In this study, we isolate and characterize CTCs through the CanPatrol system (Figure 6). Firstly, the blood sample preserved in cell preservation solution was centrifuged for 5 minutes at a speed of $1850 \mathrm{rpm}$. After removing the supernatant, the sample were mixed with phosphate buffer saline (PBS) and $4 \%$ formaldehyde for 8 minutes ${ }^{7}$. For filtration, we pass the sample through the vacuum filtration system at $0.08 \mathrm{MPa}^{7}$. This system included a filtration tube containing the membrane with 8-mm diameter pores, a vacuum pump, and a manifold vacuum plate with valve settings.

RNA-ISH was used to detect CTCs and classify CTCs into three subpopulations: mesenchymal, epithelial and mesenchymal/epithelial hybrid ${ }^{12}$ (Figure 6). The amplification process was performed in a 24-well plate. Then, we treat the samples with protease $\mathrm{K}$ and hybridize the cells with fluorescent probes specific for target sequences: red for epithelial cell adhesion molecule (EpCAM and CK8/18/19) and green for mesenchymal molecule (Vimentin and Twist) ${ }^{28}$. We used 40,6-diamidino-2- phenylindole (DAPI) to stain the nuclei, and the cells were analyzed with a fluorescent microscope (Olympus Corporation, Tokyo, Japan) ${ }^{12}$.

\section{Construction of nomogram}


The dataset was randomly divided into training and validation cohort. The selection bias refer to random classification of the two cohorts was adjusted ${ }^{29}$. T-test and Chi-square test were used to analyze the differences of clinicopathologic characteristics between two cohorts for continuous and categorical variables, respectively. The prognostic factors were determined using both univariate and multivariate analyses through the Cox's hazards regression model. Nomogram points, ranging from 1 to 100, were assigned refer to the weights for the relative importance of each model covariate determined by the final hazards regression model. In the nomogram, the total score for each patient was evaluated as a weighted sum of the contribution from each individual risk factors to predict the probability of recurrence at 1 and 2 years.

\section{Validation of nomogram}

The predictive ability of the nomogram model was measured by both discrimination and calibration. The discrimination of the nomogram model was evaluated by calibration curves, overlaying the observed probabilities and nomogram-predicted probabilities with $95 \%$ confidence interval $(95 \% \mathrm{Cl})$. As a measurement for internal validation, the Harrell's concordance index (C-index) was analyzed using 10fold cross-validation repeated for 20 times $^{30}$.

We categorized patients into three risk groups of CTC counts, M-CTC percentage and nomogram, based on the X-tile (Version 3.6.1, Yale University, New Haven, USA), a newly-developed bioinformatic tool to determine optimal cut-off points for survival analysis ${ }^{31}$. The X-tile software could tested all possible cutoff points of target quantitative data by Log-rank test and selected the lowest $p$-value and highest $\chi 2$. The EOC patients involved were then divided into three risk groups: good, intermediate and poor prognosis. In the nomogram with or without CTCs, the optimal cut-off values were 128, 251 and 98, 169. Taking CTCs into separation, the values were 5 and 9 in CTC count, 0.1 and 0.3 in the M-CTC percentage. KaplanMeier methods were used to generate the survival curves and the prognostic differences were assessed by Log-rank test. The receiver operating characteristic (ROC) curve analysis was applied to identify the prognosis value of the nomogram according to the Youden index and area under curve (AUC). All the statistical analyses were conducted by R software Version 4.0.2 (GUI 1.72 Catalina build) and graphed using Graph Prism Version 7.0a (GraphPad Software, San Diego, CA, USA). P-value $<0.05$ was defined as statistically significant.

\section{Conclusions}

CTCs, especially those undergoing EMT hold promise prognostic value as minimally-invasive biomarkers for ovarian cancer recurrence. By the advanced CanPatrol CTC-enrichment technique, our study evaluated both CTC counts and M-CTC percentage to clarify their clinical value. The prognostic nomogram based on CTCs and EMT could support clinical decision-making and provide cues for early intervention among EOC patients.

\section{Abbreviations}


CA-199, carbohydrate antigen-199; CEA , carcinoembryonic antigen; CTCs, circulating tumor cells; C-index, concordance index; DAPI, 6-diamidino-2- phenylindole; DFS, Disease-free survival; EMT, epithelialmesenchymal transition; EOC, epithelial ovarian cancer囚FIGO \International Federation of Gynecology and

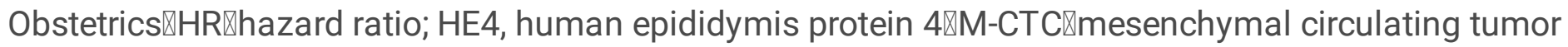

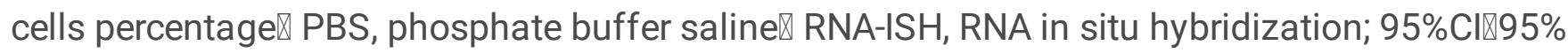
confidence interval

\section{Declarations}

Funding [This work was supported by the Three-Year Action Plan of the Program of Shanghai Hospital Development Center for Promoting Clinical Skills and Innovative Ability of Municipal Hospitals (16CR2012A).

Competing Interests[The authors declare no competing interests.

Acknowledgements $\square$ Not applicable.

Author contributions $\square$ Jiani Yang and Jun Ma performed the study and wrote the manuscript. Jiani Yang, Shan Huang and Yue jin interpreted the data and carried out statistical analysis. Shanshan Cheng and Nan Zhang collected patients' samples and clinical data. Yu Wang, Jiani Yang and Jun Ma designed the study. All authors approved the final manuscript. Jiani Yang and Jun Ma contributed equally to the work.

Data availability $[$ The data of these findings cannot be shared at this time as the data also forms part of an ongoing study. Requests for data will be considered by the corresponding author after publication of the study.

\section{References}

1 Siegel, R. L., Miller, K. D. \& Jemal, A. Cancer statistics, 2020. CA Cancer J Clin 70, 7-30, doi:10.3322/caac.21590 (2020).

2 Bray, F. et al. Global cancer statistics 2018: GLOBOCAN estimates of incidence and mortality worldwide for 36 cancers in 185 countries. CA Cancer J Clin 68, 394-424, doi:10.3322/caac.21492 (2018).

3 Jacobs, I. J. et al. Ovarian cancer screening and mortality in the UK Collaborative Trial of Ovarian Cancer Screening (UKCTOCS): a randomised controlled trial. Lancet 387, 945-956, doi:10.1016/S01406736(15)01224-6 (2016).

4 Lowes, L. E. \& Allan, A. L. Circulating Tumor Cells and Implications of the Epithelial-toMesenchymal Transition. Adv Clin Chem 83, 121-181, doi:10.1016/bs.acc.2017.10.004 (2018).

$5 \quad$ Riethdorf, S. et al. Detection of circulating tumor cells in peripheral blood of patients with metastatic breast cancer: a validation study of the CellSearch system. Clin Cancer Res 13, 920-928, 
doi:10.1158/1078-0432.CCR-06-1695 (2007).

6 Scher, H. I. et al. Circulating tumour cells as prognostic markers in progressive, castration-resistant prostate cancer: a reanalysis of IMMC38 trial data. Lancet Oncol 10, 233-239, doi:10.1016/S14702045(08)70340-1 (2009).

7 Wu, S. et al. Classification of circulating tumor cells by epithelial-mesenchymal transition markers. PLoS One 10, e0123976, doi:10.1371/journal.pone.0123976 (2015).

8 Qi, L. N. et al. Circulating Tumor Cells Undergoing EMT Provide a Metric for Diagnosis and Prognosis of Patients with Hepatocellular Carcinoma. Cancer Res 78, 4731-4744, doi:10.1158/00085472.CAN-17-2459 (2018).

9 Burinaru, T. A. et al. Detection of Circulating Tumor Cells Using Microfluidics. ACS Comb Sci 20, 107-126, doi:10.1021/acscombsci.7b00146 (2018).

10 Poveda, A. et al. Circulating tumor cells predict progression free survival and overall survival in patients with relapsed/recurrent advanced ovarian cancer. Gynecol Oncol 122, 567-572, doi:10.1016/j.ygyno.2011.05.028 (2011).

11 Allard, W. J. et al. Tumor cells circulate in the peripheral blood of all major carcinomas but not in healthy subjects or patients with nonmalignant diseases. Clin Cancer Res 10, 6897-6904, doi:10.1158/1078-0432.CCR-04-0378 (2004).

12 Chen, Y. et al. Circulating tumor cells undergoing EMT are poorly correlated with clinical stages or predictive of recurrence in hepatocellular carcinoma. Sci Rep 9, 7084, doi:10.1038/s41598-019-43572-1 (2019).

13 Guan, X. et al. The prognostic and therapeutic implications of circulating tumor cell phenotype detection based on epithelial-mesenchymal transition markers in the first-line chemotherapy of HER2negative metastatic breast cancer. Cancer Commun (Lond) 39, 1, doi:10.1186/s40880-018-0346-4 (2019).

$14 \mathrm{Kim}, \mathrm{S}$. I. et al. Development of Web-Based Nomograms to Predict Treatment Response and Prognosis of Epithelial Ovarian Cancer. Cancer Res Treat 51, 1144-1155, doi:10.4143/crt.2018.508 (2019).

15 Balachandran, V. P., Gonen, M., Smith, J. J. \& DeMatteo, R. P. Nomograms in oncology: more than meets the eye. Lancet Oncol 16, e173-180, doi:10.1016/S1470-2045(14)71116-7 (2015).

16 Gerestein, C. G. et al. The prediction of progression-free and overall survival in women with an advanced stage of epithelial ovarian carcinoma. BJOG 116, 372-380, doi:10.1111/j.14710528.2008.02033.x (2009). 
17 Bookman, M. A. Can we predict who lives long with ovarian cancer? Cancer 125 Suppl 24, 45784581, doi:10.1002/cncr.32474 (2019).

18 Budd, G. T. et al. Circulating tumor cells versus imaging-predicting overall survival in metastatic breast cancer. Clin Cancer Res 12, 6403-6409, doi:10.1158/1078-0432.CCR-05-1769 (2006).

19 Hayes, D. F. et al. Circulating tumor cells at each follow-up time point during therapy of metastatic breast cancer patients predict progression-free and overall survival. Clin Cancer Res 12, 4218-4224, doi:10.1158/1078-0432.CCR-05-2821 (2006).

20 Judson, P. L. et al. Preoperative detection of peripherally circulating cancer cells and its prognostic significance in ovarian cancer. Gynecol Onco/ 91, 389-394, doi:10.1016/j.ygyno.2003.08.004 (2003).

21 Fan, T., Zhao, Q., Chen, J. J., Chen, W. T. \& Pearl, M. L. Clinical significance of circulating tumor cells detected by an invasion assay in peripheral blood of patients with ovarian cancer. Gynecol Oncol 112, 185-191, doi:10.1016/j.ygyno.2008.09.021 (2009).

22 Guo, W. et al. Clinical significance of EpCAM mRNA-positive circulating tumor cells in hepatocellular carcinoma by an optimized negative enrichment and qRT-PCR-based platform. Clin Cancer Res 20, 4794-4805, doi:10.1158/1078-0432.CCR-14-0251 (2014).

23 Cham, S. et al. Development and validation of a risk-calculator for adverse perioperative outcomes for women with ovarian cancer. Am J Obstet Gynecol 220, 571 e571-571 e578, doi:10.1016/j.ajog.2019.02.019 (2019).

24 Duska, L. R., Java, J. J., Cohn, D. E. \& Burger, R. A. Risk factors for readmission in patients with ovarian, fallopian tube, and primary peritoneal carcinoma who are receiving front-line chemotherapy on a clinical trial (GOG 218): an NRG oncology/gynecologic oncology group study (ADS-1236). Gynecol Oncol 139, 221-227, doi:10.1016/j.ygyno.2015.08.011 (2015).

25 Lee, C. K. et al. Development and validation of a prognostic nomogram for overall survival in patients with platinum-resistant ovarian cancer treated with chemotherapy. Eur J Cancer 117, 99-106, doi:10.1016/j.ejca.2019.05.029 (2019).

26 Bogani, G. et al. A score system for complete cytoreduction in selected recurrent ovarian cancer patients undergoing secondary cytoreductive surgery: predictors- and nomogram-based analyses. $J$ Gynecol Oncol 29, e40, doi:10.3802/jgo.2018.29.e40 (2018).

27 Adams, D. L., Alpaugh, R. K., Tsai, S., Tang, C. M. \& Stefansson, S. Multi-Phenotypic subtyping of circulating tumor cells using sequential fluorescent quenching and restaining. Sci Rep 6, 33488, doi:10.1038/srep33488 (2016).

28 Zhao, X. H. et al. Molecular detection of epithelial-mesenchymal transition markers in circulating tumor cells from pancreatic cancer patients: Potential role in clinical practice. World J Gastroentero/ 25, 
29 Iasonos, A., Schrag, D., Raj, G. V. \& Panageas, K. S. How to build and interpret a nomogram for cancer prognosis. J Clin Oncol 26, 1364-1370, doi:10.1200/JC0.2007.12.9791 (2008).

30 Harrell, F. E., Jr., Lee, K. L. \& Mark, D. B. Multivariable prognostic models: issues in developing models, evaluating assumptions and adequacy, and measuring and reducing errors. Stat Med 15, 361387, doi:10.1002/(SICI)1097-0258(19960229)15:4<361::AID-SIM168>3.0.C0;2-4 (1996).

31 Camp, R. L., Dolled-Filhart, M. \& Rimm, D. L. X-tile: a new bio-informatics tool for biomarker assessment and outcome-based cut-point optimization. Clin Cancer Res 10, 7252-7259, doi:10.1158/1078-0432.CCR-04-0713 (2004).

\section{Figures}
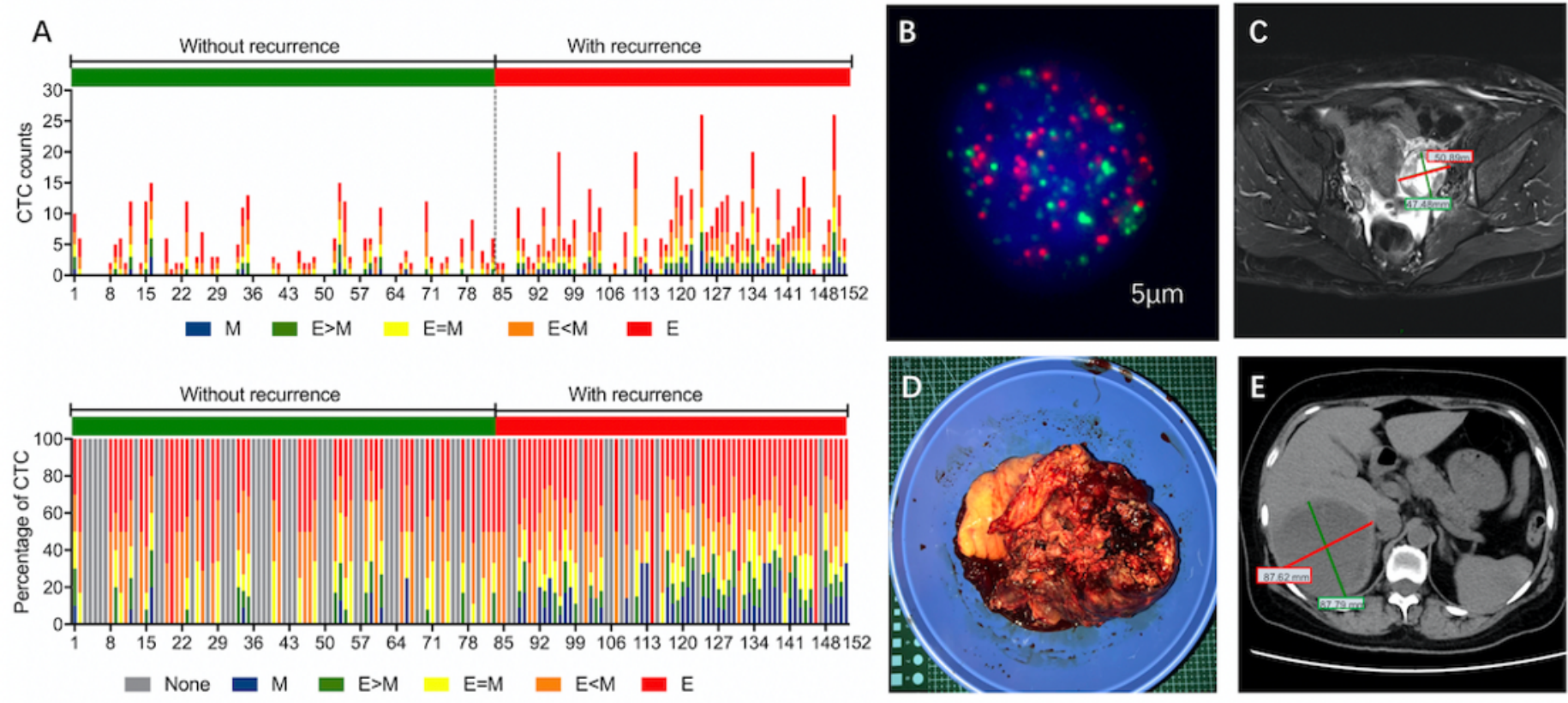

\section{Figure 1}

(A) Total CTC counts (top) and percentage of each CTC categories (bottom) before treatment in ovarian cancer patients with or without recurrence. (B) The CTC image and (C) Magnetic Resonance Imaging (MRI) image of an representative patient who had CTC-positive blood sample before treatment. (D) After tumor resection, the patient suffered cancer recurrence with (E) detectable liver metastasis observed by Magnetic Resonance Imaging (MRI) at 6-month follow-up. 
A

Point

FIGO stage

Pathological grade

Lymph node metastasis

Ascites

M-CTC percentage

CTC counts

CA-125

Total points

1-year recurrence rate

2-year recurrence rate

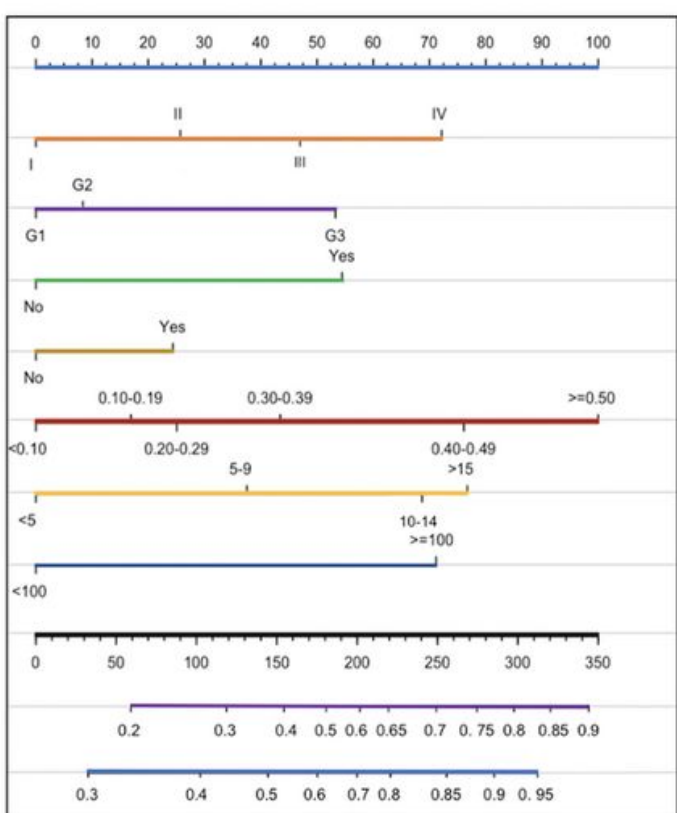

B

Point

FIGO stage

Pathological grade

Lymph node metastasis

Ascites

CA-125

Total points

1-year recurrence rate

2-year recurrence rate

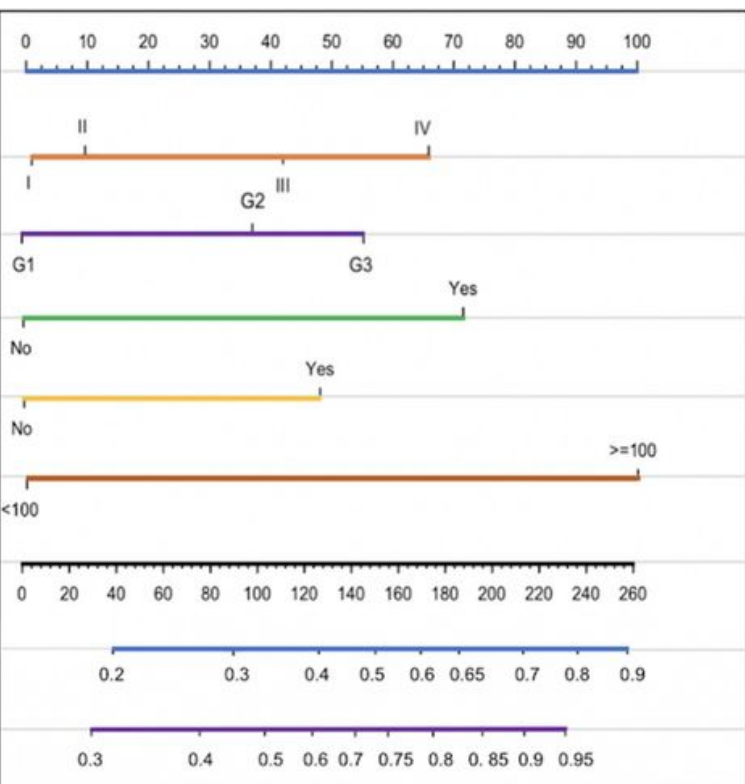

\section{Figure 2}

The nomogram models 1-year recurrence rate and 2-year recurrence rate of ovarian cancer patients $(A)$ based on CTC counts and M-CTC percentage; (B) without CTC counts and M-CTC percentage 
A

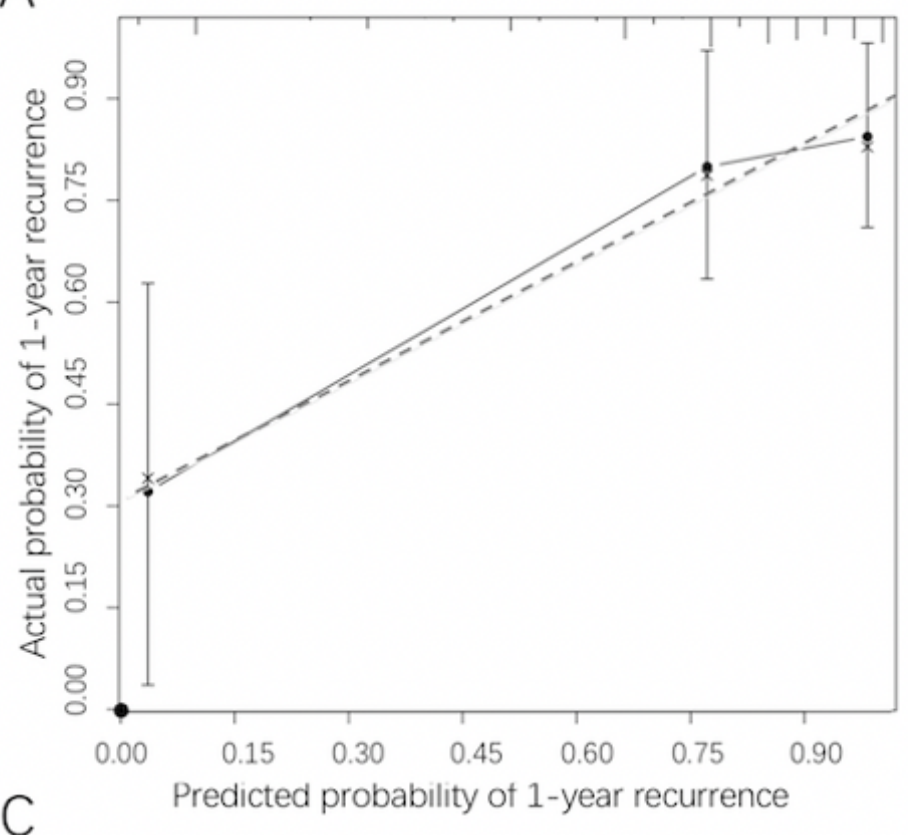

C

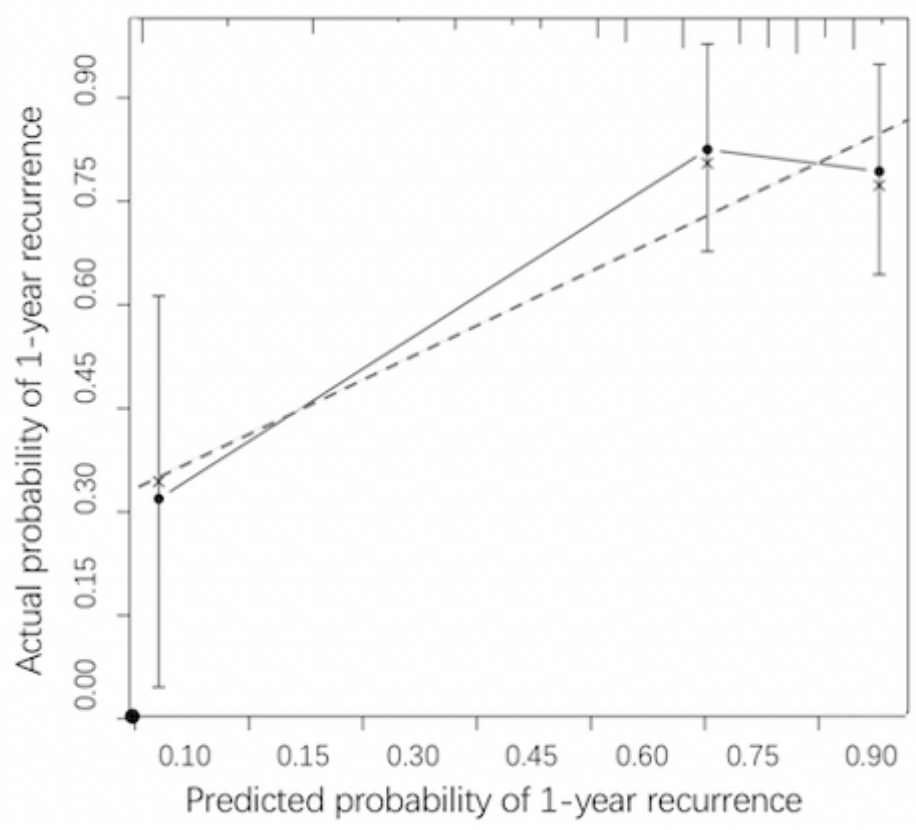

B
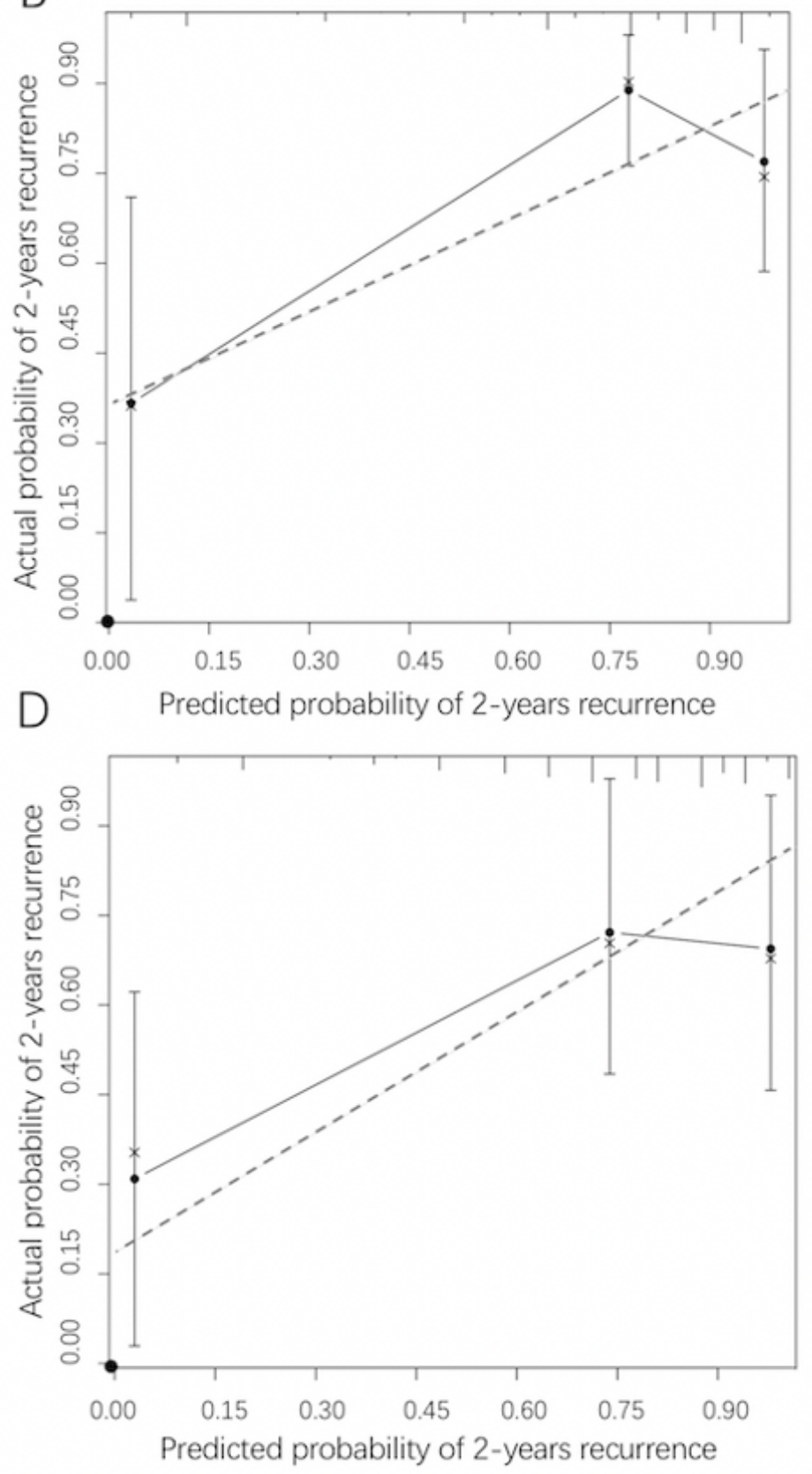

Figure 3

The calibration curves of internal (A, B) and external (C, D) validation of the nomogram constructed in the training group based on CTCs count and M-CTC percentage. The predicted probabilities of 1-year and 2year recurrence were consistent with the actual recurrence proportions of ovarian cancer patients. 

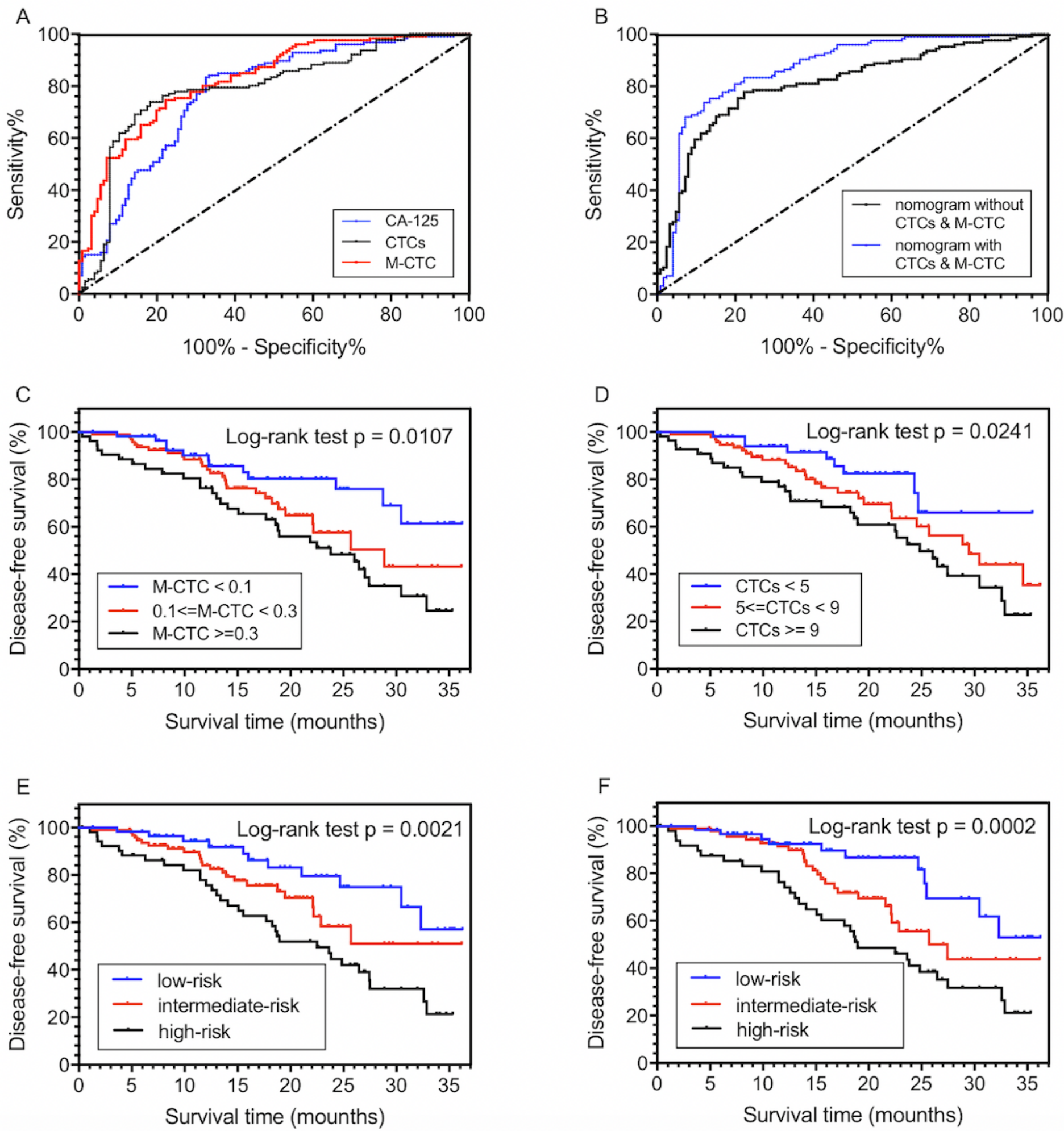

\section{Figure 4}

The receive operating characteristic (ROC) curve of patients stratified by (A) CTC counts, M-CTC percentage, and CA-125; (B) nomogram-based risk groups The Kaplan-Meier curves for DFS of all the patients involved stratified by (C) M-CTC percentage; (D) CTC counts and risk groups of the nomogram (E) without or $(\mathrm{F})$ with $\mathrm{CTC}$ counts and M-CTC percentage. 


\section{1 patients with pathologically diagnosed}

EOC were enrolled

9 patients were excluded

- underwent other treatments, such as neoadjuvant therapy, radiotherapy, or immunotherapy $(n=5)$

- without consent to use medical information for the research purpose $(n=4)$

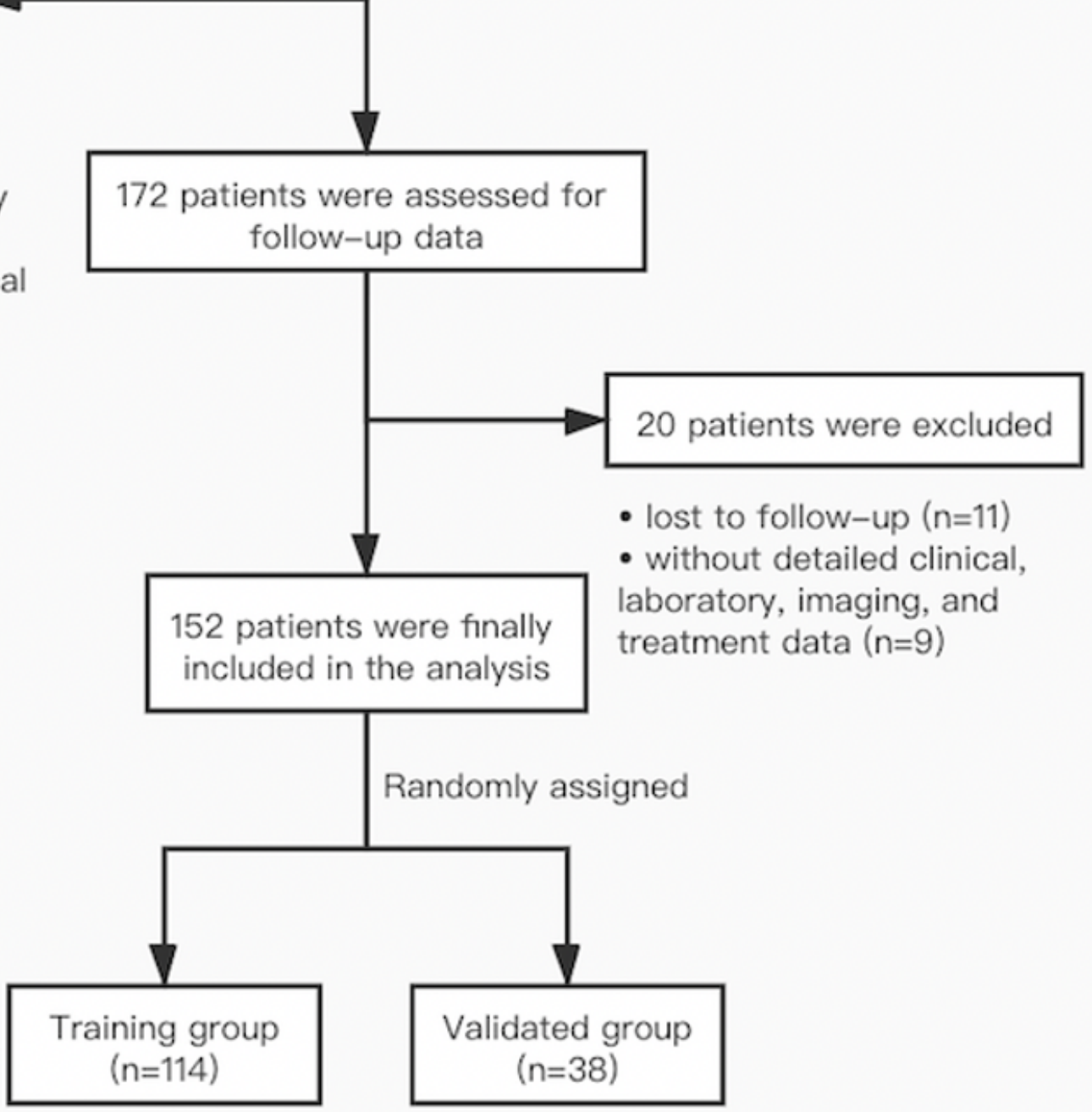

\section{Figure 5}

Patient enrollment flow chart 

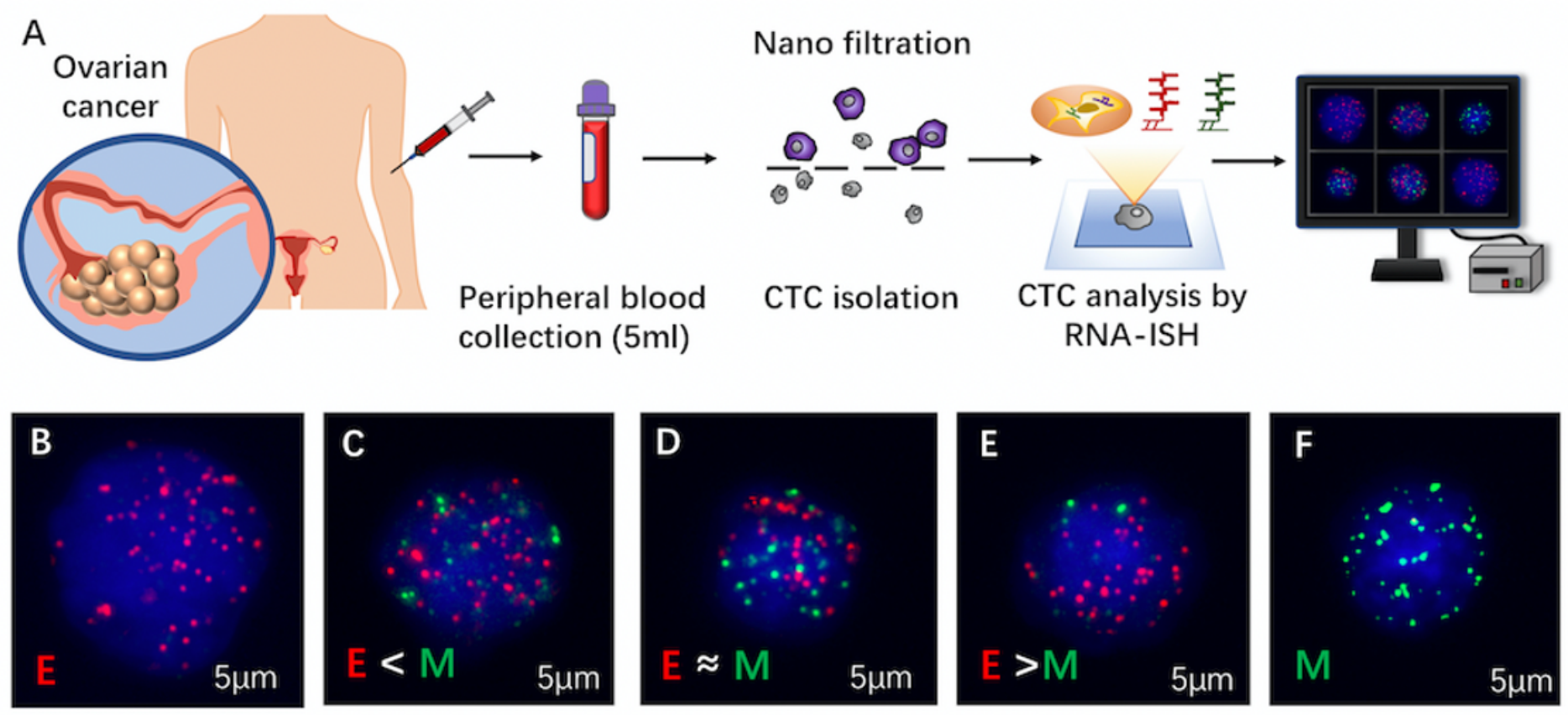

Figure 6

(A) Process of circulating tumor cells (CTCs) isolation and detection by CanPatrol CTC enrichment and ISH. (B-F) 5 representative images of patients with different CTC subpopulations, based on the RNA-ISH of mesenchymal (vimentin and Twist, green fluorescence) and epithelial (EpCAM and CK8/18/19, red fluorescence) markers. 